Collection SFN 7 (2007) 1-11

(c) EDP Sciences, Les Ulis

DOI: $10.1051 / \mathrm{sfn}: 2007016$

\title{
La polarisation des neutrons : Qu'est-ce que c'est ? à quoi ça sert?
}

\section{J. Schweizer ${ }^{1}$}

${ }^{1}$ DRFMC/MDN, CEA-Grenoble, 17 rue des Martyrs, 38054 Grenoble Cedex 9, France

\section{INTRODUCTION : LA RÈGLE D'OR DE FERMI}

Pour exprimer la section efficace de diffusion neutronique on utilise habituellement la règle d'or de Fermi qui évalue les probabilités de transition. On considère la transition où le système diffuseur passe d'un état $|\lambda\rangle$ à un état $\left|\lambda^{\prime}\right\rangle$ et où l'état du neutron passe de $|\mathbf{k}\rangle$ à $\left|\mathbf{k}^{\prime}\right\rangle$, $\mathbf{k}$ étant le vecteur d'onde du neutron. La section efficace de diffusion est alors proportionnelle à cette probabilité :

$$
\left(\frac{d^{2} \sigma}{d \Omega d E^{\prime}}\right)_{\substack{\mathbf{k} \rightarrow \mathbf{k}^{\prime} \\ \lambda \rightarrow \lambda^{\prime}}}=\frac{k^{\prime}}{k}\left(\frac{m}{2 \pi \hbar^{2}}\right)^{2}\left|\left\langle\mathbf{k}^{\prime}, \lambda^{\prime}|V| \mathbf{k}, \lambda\right\rangle\right|^{2} \delta\left(E^{\prime}+E_{\lambda^{\prime}}-E-E_{\lambda}\right)
$$

Une telle expression n'est pourtant pas complète. Elle devrait être remplacée par une expression similaire, mais où le neutron n'est pas seulement caractérisé par son vecteur d'onde $|\mathbf{k}\rangle$ mais aussi par son spin :

$$
\left(\frac{d^{2} \sigma}{d \Omega d E^{\prime}}\right)_{\substack{\mathbf{k} \rightarrow \mathbf{k}^{\prime} \\ \lambda \rightarrow \lambda^{\prime} \\ \chi \rightarrow \chi^{\prime}}}=\frac{k^{\prime}}{k}\left(\frac{m}{2 \pi \hbar^{2}}\right)^{2}\left|\left\langle\mathbf{k}^{\prime}, \lambda^{\prime}, \chi^{\prime}|V| \mathbf{k}, \lambda, \chi\right\rangle\right|^{2} \delta\left(E^{\prime}+E_{\lambda^{\prime}}-E-E_{\lambda}\right)
$$

En effet, le neutron possède un spin $\mathbf{S}$ et l'état de ce spin est représenté par la fonction d'onde de spin $|\chi\rangle$. Les spins des neutrons peuvent être polarisés et cette polarisation est un atout important en diffusion de neutrons.

\section{LE SPIN DU NEUTRON ET SA POLARISATION}

\subsection{Le neutron possède un spin $S$}

Le neutron possède un spin $\mathbf{S}$, qui est un moment angulaire interne de nombre quantique $S=\frac{1}{2}$. Cette valeur $S=\frac{1}{2}$ veut dire deux choses :

- Si on mesure la valeur prise par l'opérateur $\mathbf{S}^{2}$, on ne peut trouver qu'une seule valeur, la valeur propre : $S(S+1) \hbar^{2}=\frac{3}{4} \hbar^{2}$

- Si on mesure la valeur prise par l'opérateur $S_{z}$, projection de l'opérateur $\mathbf{S}$ sur l'axe Oz, on ne peut trouver qu'une des deux valeurs propres : ou bien $m_{s}=+\frac{1}{2} \hbar$, ou bien $m_{s}=-\frac{1}{2} \hbar$, et ceci quelque soit l'axe Oz choisi.

\subsection{La fonction d'onde de spin $\chi$}

On peut définir une fonction d'onde de spin, c'est à dire une fonction d'onde dans l'espace des spins, qui est une combinaison linéaire des deux états : 
- $|+\rangle: S_{z}$ dirigé selon $\mathrm{Oz}$

- $|-\rangle: S_{z}$ dirigé selon $-\mathrm{Oz}$

$$
|\chi\rangle=a|+\rangle+b|-\rangle
$$

avec $|a|^{2}+|b|^{2}=1$.

\subsection{Les matrices de Pauli}

$\mathbf{S}$ est un opérateur vectoriel, c'est à dire un opérateur à 3 composantes. Les représentations de ces composantes sont les 3 matrices :

$$
\begin{aligned}
S_{x} & =\frac{\hbar}{2}\left(\begin{array}{cc}
0 & 1 \\
1 & 0
\end{array}\right) \\
S_{y} & =\frac{\hbar}{2}\left(\begin{array}{rr}
0 & -\imath \\
\imath & 0
\end{array}\right) \\
S_{z} & =\frac{\hbar}{2}\left(\begin{array}{rr}
1 & 0 \\
0 & -1
\end{array}\right)
\end{aligned}
$$

Par la suite, nous ne nous encombrerons plus du facteur $\hbar / 2$ et, à la place de l'opérateur de spin $\mathbf{S}$, nous utiliserons l'opérateur $\boldsymbol{\sigma}=2 \mathbf{S} / \hbar$, opérateur vectoriel dont les 3 composantes sont les 3 matrices de Pauli :

$$
\begin{aligned}
\sigma_{x} & =\left(\begin{array}{ll}
0 & 1 \\
1 & 0
\end{array}\right) \\
\sigma_{y} & =\left(\begin{array}{rr}
0 & -\imath \\
\imath & 0
\end{array}\right) \\
\sigma_{z} & =\left(\begin{array}{rr}
1 & 0 \\
0 & -1
\end{array}\right)
\end{aligned}
$$

\subsection{Valeurs moyennes et polarisation}

Partons d'une fonction d'onde de spin pour un neutron :

$$
|\chi\rangle=a|+\rangle+b|-\rangle
$$

$\mathrm{Si}$ on mesure la valeur prise par la projection $\sigma_{z}$ selon $\mathrm{Oz}$, on trouve ou bien +1 ou bien -1 , mais certainement pas une autre valeur. Si on réalise la même mesure sur un grand nombre de neutrons qui ont tous la même fonction d'onde de spin (2.1), lorsque l'on fait la moyenne sur les +1 et les -1 que l'on a trouvés, on arrive à la 'valeur moyenne' :

$$
\begin{gathered}
\left\langle\sigma_{z}\right\rangle=\left\langle\chi\left|\sigma_{z}\right| \chi\right\rangle \\
\left\langle\sigma_{z}\right\rangle=\left\langle\left( a^{*}\left\langle+\left|+b^{*}\langle-|\right)\left|\sigma_{z}\right|(a|+\rangle+b|-\rangle)\right\rangle\right.\right. \\
\left\langle\sigma_{z}\right\rangle=\left(a^{*}, b^{*}\right)\left(\begin{array}{rr}
1 & 0 \\
0 & -1
\end{array}\right)\left(\begin{array}{l}
a \\
b
\end{array}\right)=\left(a^{*}, b^{*}\right)\left(\begin{array}{r}
a \\
-b
\end{array}\right) \\
\left\langle\sigma_{z}\right\rangle=a a^{*}-b b^{*}=|a|^{2}-|b|^{2}
\end{gathered}
$$

Si le neutron est dans l'état $|+\rangle$, c'est à dire que $a=1$ and $b=0$, alors $\left\langle\sigma_{z}\right\rangle=1$. Si au contraire le neutron est dans l'état $|-\rangle$, avec $a=0$ et $b=1$, alors $\left\langle\sigma_{z}\right\rangle=-1$. 
Dans le cas général où a et $\mathrm{b}$ sont tous les deux différents de zéro, $\left\langle\sigma_{z}\right\rangle=|a|^{2}-|b|^{2}$.

De la même façon, si on mesure la composante $x$ de l'opérateur $\sigma$, on trouve là encore soit +1 , soit -1 . Mais pour un grand nombre de neutrons qui ont tous la même fonction d'onde de spin (2.1), la valeur moyenne des mesures est :

$$
\begin{aligned}
& \left\langle\sigma_{x}\right\rangle=\left\langle\chi\left|\sigma_{x}\right| \chi\right\rangle=\left\langle\chi\left|\left(\begin{array}{cc}
0 & 1 \\
1 & 0
\end{array}\right)\right| \chi\right\rangle \\
& \left\langle\sigma_{x}\right\rangle=a^{*} b+a b^{*}=2 \Re\left(a^{*} b\right)
\end{aligned}
$$

et la valeur moyenne selon Oy est :

$$
\begin{aligned}
& \left\langle\sigma_{y}\right\rangle=\left\langle\chi\left|\sigma_{y}\right| \chi\right\rangle=\left\langle\chi\left|\left(\begin{array}{rr}
0 & -\imath \\
\imath & 0
\end{array}\right)\right| \chi\right\rangle \\
& \left\langle\sigma_{y}\right\rangle=\imath\left(a b^{*}-a^{*} b\right)=2 \Im\left(a^{*} b\right)
\end{aligned}
$$

Les 3 quantités $\left\langle\sigma_{x}\right\rangle,\left\langle\sigma_{y}\right\rangle$ et $\left\langle\sigma_{z}\right\rangle$ sont les 3 composantes du vecteur $\mathbf{p}$, 'polarisation du neutron' :

$$
\mathbf{p}\left\{\begin{array}{l}
p_{x}=\left\langle\sigma_{x}\right\rangle \\
p_{y}=\left\langle\sigma_{y}\right\rangle \\
p_{z}=\left\langle\sigma_{z}\right\rangle
\end{array}\right.
$$

Nota : L'opérateur de spin $\mathbf{S}$, ainsi que l'opérateur 'matrices de Pauli' $\boldsymbol{\sigma}=2 \mathbf{S} / \hbar$ sont des opérateurs vectoriels quantiques : il n'est pas possible de connaître en même temps leurs 3 composantes. Il n'en est pas de même de l'opérateur polarisation $\mathbf{p}$ qui est un vecteur classique dans la mesure où il est défini à partir de 3 valeurs moyennes. En ce qui le concerne, il est donc possible :

- de mesurer en même temps ses 3 composantes

- de contrôler sa rotation dans l'espace à 3 dimensions

\subsection{Exemples de fonctions d'onde de spin et de polarisation d'un neutron} Prenons l'exemple de la fonction d'onde de spin $|\chi\rangle=\frac{1}{\sqrt{2}}[|+\rangle+|-\rangle]$. Comme $a=b=\frac{1}{\sqrt{2}}$, nous
avons :

$$
\begin{aligned}
& \left\langle\sigma_{x}\right\rangle=\left\langle\chi\left|\sigma_{x}\right| \chi\right\rangle=a b^{*}+a^{*} b=1 \\
& \left\langle\sigma_{y}\right\rangle=\left\langle\chi\left|\sigma_{y}\right| \chi\right\rangle=\imath\left(a b^{*}-a^{*} b\right)=0 \\
& \left\langle\sigma_{z}\right\rangle=\left\langle\chi\left|\sigma_{z}\right| \chi\right\rangle=|a|^{2}-|b|^{2}=0
\end{aligned}
$$

Lorsque on mesure la composante $\sigma_{z}$ d'un ensemble de neutrons ayant cette fonction d'onde de spin, des fois on trouve +1 et des fois -1 , mais la moyenne de ces mesures est 0 . Lorsque on mesure la composante $\sigma_{y}$ de cet ensemble de neutrons, là aussi on trouve soit +1 soit -1 , avec une moyenne de 0 . Mais lorsque on mesure la composante $\sigma_{x}$ de ce même ensemble de neutrons, on trouve toujours +1 . Ces neutrons sont polarisés selon $\mathbf{O x}$.

De la même façon, avec une fonction d'onde de spin : $|\chi\rangle=\frac{1}{\sqrt{2}}[|+\rangle-|-\rangle]$, la polarisation est selon $-\mathbf{O x}$.

Avec une fonction d'onde de spin : $|\chi\rangle=\frac{1}{\sqrt{2}}[|+\rangle+\imath|-\rangle]$, la polarisation est selon $\mathbf{O y}$.

Avec une fonction d'onde de spin : $|\chi\rangle=\frac{1}{\sqrt{2}}[|+\rangle-\imath|-\rangle]$, la polarisation est selon $-\mathbf{O y}$.

Nota : A partir des exemples précédents, on voit dans l'expression des fonctions d'onde de spin que la phase qui existe entre $|+\rangle$ et $|-\rangle$ détermine la direction de polarisation dans le plan $x y$. 


\subsection{Polarisation d'un faisceau de neutrons}

Dans un faisceau de neutrons, les polarisations individuelles de chaque neutron peuvent différer les unes des autres. On définit la polarisation du faisceau comme la moyenne des polarisations individuelles de tous les neutrons du faisceau :

$$
\mathbf{P}=\frac{1}{N} \sum_{j} \mathbf{p}_{\mathbf{j}}
$$

avec, bien entendu :

$$
0 \leq|\mathbf{P}| \leq 1
$$

\section{SPIN ET MOMENT MAGNÉTIQUE DU NEUTRON}

\subsection{Le neutron a un moment magnétique}

Le neutron a un moment magnétique et ce moment est relié au moment angulaire de spin par :

$$
\boldsymbol{\mu}_{\boldsymbol{n}}=\gamma_{L}\langle\boldsymbol{S}\rangle
$$

Pour le neutron le rapport gyromagnétique $\gamma_{L}$ est négatif, ce qui signifie que le moment magnétique est opposé au moment de spin.

\subsection{Action d'un champ magnétique}

Un champ magnétique exerce un couple sur le moment magnétique du neutron, comme il le fait sur n’importe quel moment magnétique :

$$
\boldsymbol{\Gamma}=\boldsymbol{\mu}_{\boldsymbol{n}} \times \boldsymbol{B}=\gamma_{L}\langle\boldsymbol{S}\rangle \times \boldsymbol{B}
$$

Les champs magnétiques sont donc des outils fréquemment utilisés pour contôler la polarisation des neutrons.

Dans un champ magnétique constant, la précession de Larmor

Comparons l'action d'un champ magnétique sur le moment magnétique du neutron à l'action de la gravité sur une toupie. Le champ de gravité exerce une force, le poids $\mathbf{w}$, qui s'applique au centre de gravité de la toupie (voir Figure 1), et qui conduit L un couple :

$$
\Gamma=\boldsymbol{O G} \times \boldsymbol{w}
$$

Si la toupie ne tourne pas, ce couple va avoir comme effet de faire tomber la toupie. Mais si la toupie tourne, l'axe de la toupie (le moment angulaire $\ell$ ) va tourner autour de l'axe vertical, c'est à dire la direction du champ de gravitation.

De la même façon, dans un champ magnétique $\mathrm{B}$, un couple est exercé sur le moment magnétique du neutron (formule 3.2), et ce moment (donc la polarisation) tourne autour du champ magnétique.

La pulsation associée (pulsation de Larmor) est donnée par :

$$
\omega_{L}=\gamma_{L} B
$$

qui correspond Ĺ une rotation très rapide : plus de 3000 tours/sec dans un champ de 1 gauss et $10^{4}$ fois plus vite dans un champ de 1 tesla. 


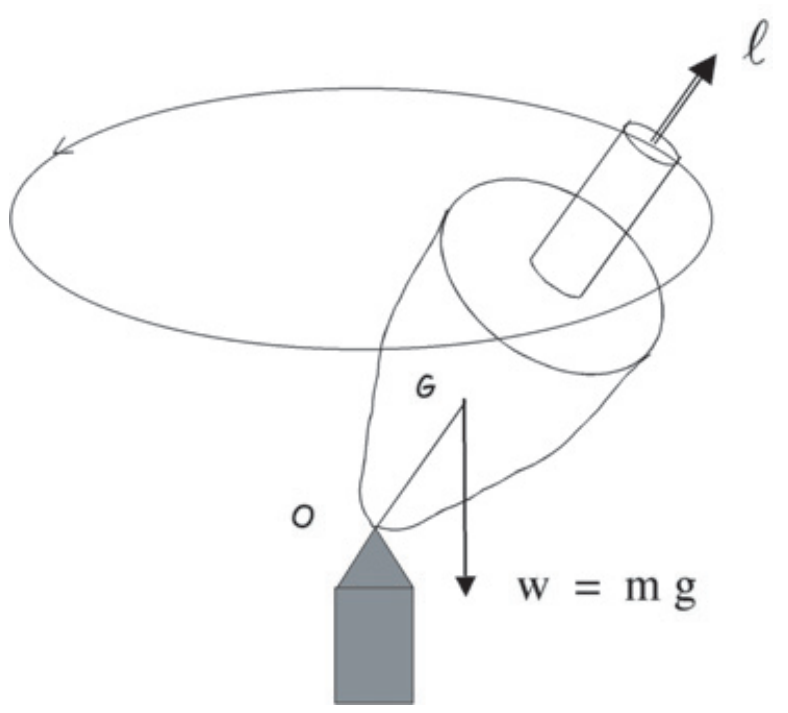

Figure 1. Le moment angulaire d'une toupie tournant autour de l'axe de gravitation.

Dans un champ magnétique variable

La polarisation ne cesse pas de tourner autour du champ $B$ avec la fréquence de Larmor $2 \pi \omega_{L}$. Qu'arrivet-il quand la direction du champ champ varie le long du trajet des neutrons? Nous allons considérer deux cas extrêmes.

- Si la direction du champ varie lentement par rapport Ĺ la fréquence de Larmor la polarisation du neutron fait de nombreux tours autour du champ pendant que le champ effectue un angle infime. Le neutron ne sent pas réellement que le champ tourne et sa polarisation suit le champ en tournant autour de lui pendant toute la variation. Une telle variation est dite 'adiabatique'.

- Si le champ varie brutalement par rapport $\mathbf{L}$ la fréquence de Larmor, la polarisation du neutron n'a pas le temps de réagir quand la direction du champ change : elle tournait autour d'un champ $B_{1}$ et soudain, bien plus rapidement qu'une période de Larmor, le champ $B_{1}$ devient $B_{2}$. Pris au dépourvu, le neutron cesse de tourner autour de $B_{1}$ qui n'existe plus et se met à tourner autour de $B_{2}$.

Dans les cas intermédiaires, quand la vitesse angulaire $\omega_{B}$ avec laquelle tourne le champ est du même ordre de grandeur que la pulsation de Larmor, la rotation de la polarisation n'est que partielle et dépend du paramètre d'adiabaticité $\omega_{L} / \omega_{B}$.

\section{Champs guides}

Dans la pratique, une fois les neutrons polarisés par le dispositif adéquat, n'importe quel champ magnétique qui se trouve sur leur passage (champ de fuite, champ terrestre...) est susceptible de faire tourner leur polarisation de manière incontrollée, donc de dépolariser les neutrons. Pour remédier Ĺ cet inconvénient, un champ magnétique est installé tout le long du passage des neutrons afin de jouer le rôle de guide. En effet, la polarisation tourne autour de ce champ ; si un champ parasite existe il modifiera légèrement la direction du champ guide, mais la polarisation des neutrons continuera de tourner autour du champ-guide qui contrôle ainsi la polarisation du faisceau. 


\section{DIFFUSION DE NEUTRONS POLARISÉS}

\subsection{Neutrons polarisés sans analyse de polarisation $[1,2]$}

\section{Dispositif expérimental}

Pour le plus simple des diffractomètres à neutrons polarisés, les neutrons incidents sont polarisés, mais la polarisation des neutrons diffusés n'est pas analysée (voir Figure 2).

Le monochromateur joue un double rôle : sélectionner des neutrons d'une seule longueur d'onde et polariser leur spin. Pour réaliser ce dernier point, le monochromateur doit être mono-domaine, ce qui est obtenu grâce à un champ magnétique vertical. La polarisation du faisceau incident peut être renversée par un dispositif de flipping qui se trouve entre le monochromateur et l'échantillon, permettant aux neutrons incidents d'arriver sur l'échantillon avec au choix une polarisation 'up' ou 'down', c'est à dire parallèle ou antiparallèle au champ magnétique qui est appliqué sur l'échantillon.

Des champs guides verticaux sont là pour maintenir la polarisation tout au long du trajet entre le monochromateur et l'échantillon, que le dispositif de flipping soit activé ou non.

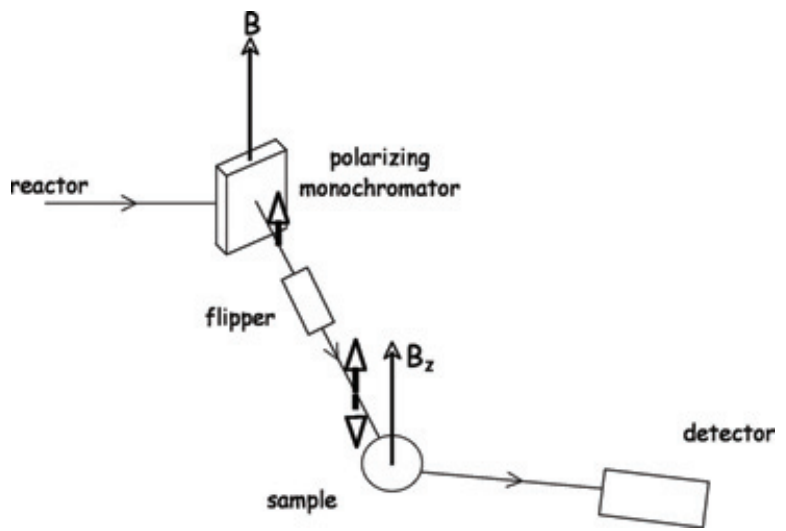

Figure 2. Schéma d'un diffractomètre à neutrons polarisés sans analyse de polarisation.

Une expérience consiste à mesurer deux fois chaque réflexion de Bragg, une avec la polarisation incidente parallèle à l'aimantation de l'échantillon $\left(I^{+}\right)$, et l'autre avec la polarisation incidente antiparallèle à cette aimantation $\left(I^{-}\right)$.

Une sensibilité décuplée

Considérons une réflexion de Bragg qui est due à la fois à une diffusion nucléaire d'amplitude $N$ et à une diffusion magnétique d'amplitude $M$. Si les neutrons ne sont pas polarisés, l'intensité mesurée est la somme de l'intensité nucléaire et de l'intensité magnétique ${ }^{1}$ :

$$
I=N^{2}+M^{2}
$$

Pour la même réflexion de Bragg, si les neutrons incidents sont polarisés dans la direction + (ou -), l'intensité mesurée est essentiellement le carré de la somme (ou de la différence) de l'amplitude nucléaire et de l'amplitude magnétique :

$$
I^{ \pm}=(N \pm M)^{2}=N^{2}+M^{2} \pm 2 N M
$$

${ }^{1}$ Pour simplifier l'écriture, nous avons fait figurer dans les formules des carrés au lieu de carrés de modules 
Cette expression représente, par rapport à la précédente, une formidable amélioration de la sensibilité lorsque l'on mesure de petites amplitudes magnétiques. Pour illustrer cette sensibilité, prenons un exemple où l'amplitude magnétique est égale à $10 \%$ de l'amplitude nucléaire $(M=0.1 N)$. Pour des neutrons non polarisés, l'intensité magnétique ne représente que $1 \%$ de l'intensité nucléaire $\left(N^{2}+M^{2}=\right.$ $1+0.01)$. Pour des neutrons polarisés la contribution magnétique représente environ $20 \%$ de la contribution nucléaire $\left(N^{2}+M^{2} \pm 2 N M=1+0.01 \pm 0.20\right)$. Cette augmentation de sensibilité est due au double produit $N M$ : l'amplitude nucléaire $N$ sert d'amplificateur pour la mesure de $M$.

Grâce aux neutrons polarisés, sans avoir à mettre en oeuvre une analyse de la polarisation, il va être possible de mesurer avec précision de petites amplitudes magnétiques et, en particulier, celles des réflexions de Bragg qui se trouvent loin dans l'espace réciproque. On a là l'outil de base pour l'étude des facteurs de forme magnétiques et des cartes de densité de spin (ou d'aimantation).

\section{Chiralité}

La formule 4.2 telle qu'elle a été écrite plus haut est incomplète car elle a oublié un terme. Une expression plus correcte aurait été :

$$
I^{ \pm}=N^{2}+M^{2} \pm 2\left[N M_{z}+\imath\left(\boldsymbol{M}^{*} \times \boldsymbol{M}\right)_{z}\right]
$$

où $O z$ est la direction de la polarisation incidente. Cette formule inclut la contribution chirale $\imath\left(\boldsymbol{M}^{*} \times \boldsymbol{M}\right)$, terme qui disparait lorsque les neutrons ne sont pas polarisés.

D'où vient un tel terme? Pour le savoir, décomposons l'amplitude magnétique $\mathbf{M}$ en partie réelle et partie imaginaire :

Alors

$$
\begin{aligned}
\boldsymbol{M} & =\mathbf{A}+\imath \mathbf{B} \\
\boldsymbol{M}^{*} & =\mathbf{A}-\imath \mathbf{B} \\
\imath\left(\boldsymbol{M}^{*} \times \boldsymbol{M}\right) & =2 \mathbf{B} \times \mathbf{A}
\end{aligned}
$$

Une structure magnétique est chirale si elle n'est pas superposable à son image dans un miroir (comme une main droite ou une main gauche). Alors $\boldsymbol{M}$ n'est pas parallèle à $\boldsymbol{M}^{*}$, ou, ce qui est équivalent, si $\mathbf{A}$ n'est pas parallèle Ĺ B. Rappelons ici que l'amplitude magnétique $\boldsymbol{M}$ est, pour une structure cristalline, égale au facteur de structure magnétique, et que tous les deux sont des vecteurs.

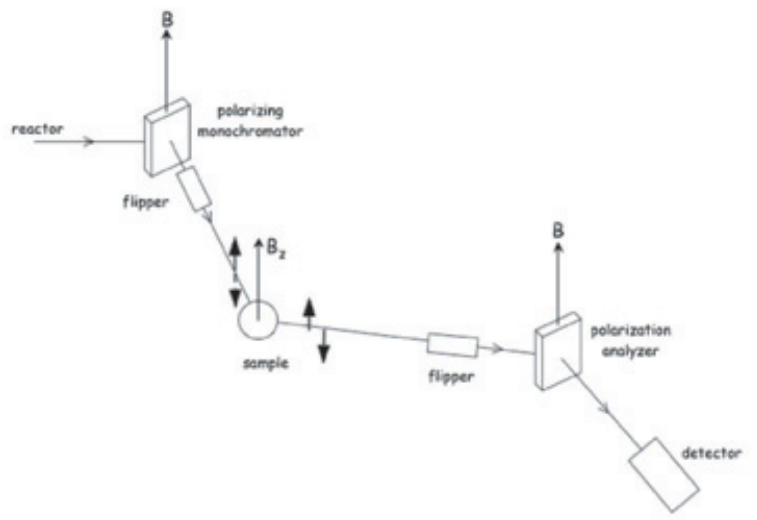

Figure 3. Schéma de principe pour l'analyse de polarisation uniaxiale [3].

L'exemple le plus simple de structure chirale est l'hélice où, avec un atome par maille $\boldsymbol{M}=\mathbf{u}+\imath \mathbf{v}$. Mais une structure magnétique très générale, avec plusieurs moments non colinéaires par maille, peut 
très bien avoir des facteurs de structure magnétiques qui ne sont pas parallèles à leur conjugués. De telles structures sont chirales et cette chiralité peut être analysée avec des neutrons polarisés.

\subsection{Analyse de polarisation uniaxiale (longitudinale) [3]}

\section{Description de la méthode}

Moon et al. [3] ont ouvert un nouveau domaine de recherche en montrant que l'on gagnait beaucoup d'informations en analysant la polarisation des neutrons diffusés, grâce à un analyseur placé derrière l'échantillon.

Dans cette première version de l'analyse de polarisation, les neutrons incidents sont polarisés dans une certaine direction de l'espace, et la polarisation des neutrons diffusés est analysée dans la même direction de l'espace (voir Figure 3). C'est pourquoi cette méthode d'analyse est appelée analyse de polarisation uniaxiale. Il arrive que l'appellation 'analyse de polarisation longitudinale' soit aussi utilisée.

Les 4 intensités (sections efficaces) partielles

En introduisant aussi un deuxième flipper entre l'échantillon et l'analyseur de polarisation, il est possible de sélectionner non seulement la direction + ou - pour la polarisation incidente, mais aussi la direction + ou - pour la polarisation diffusée. Il est alors possible de mesurer 4 intensités (sections efficaces) partielles correspondant aux 4 combinaisons ${ }^{2}$ :

$$
\begin{aligned}
& I^{++} \propto \sigma^{++}=\left(N+M_{z}\right)^{2} \\
& I^{--} \propto \sigma^{--}=\left(N-M_{z}\right)^{2} \\
& I^{+-} \propto \sigma^{+-}=\left(M_{x}+\imath M_{y}\right)^{2} \\
& I^{-+} \propto \sigma^{-+}=\left(M_{x}-\imath M_{y}\right)^{2}
\end{aligned}
$$

Les 2 sections efficaces partielles $\sigma^{++}$et $\sigma^{--}$correspondent à des situations où le spin du neutron reste le même pendant le processus de diffusion. On les appelle les sections efficaces 'non spin flip'. Elles concernent l'amplitude nucléaire et la composante de l'amplitude magnétique qui est parallèle L la polarisation. Au contraire, $\sigma^{+-}$et $\sigma^{-+}$sont des sections efficaces 'spin flip' et représentent une diffusion qui n'est pas due à l'amplitude nucléaire, mais n'est due qu'aux composantes magnétiques perpendiculaires à la polarisation.

Comme cela va être développé dans les autres cours de cette école, l'analyse de polarisation uniaxiale est devenu la méthode de base pour séparer la diffusion magnétique de la diffusion nucléaire lorsque ces deux diffusions ont lieu dans une même région de l'espace réciproque.

Analyse de polarisation uniaxiale : la méthode $X Z$

Les formules (4.4-4.7) sont incomplètes dans la mesure où on $y$ a omis la diffusion incohérente (incohérent isotopique $N N_{\text {incoh }}$ et incohérent de spin nucléaire $\left.S S_{\text {incoh }}\right)$. Si on tient compte de ces contributions, les formules complètes sont :

$$
\begin{aligned}
& \sigma^{++}=\left(N+M_{z}\right)^{2}+\frac{1}{3} S S_{\mathrm{incoh}}+N N_{\mathrm{incoh}} \\
& \sigma^{--}=\left(N-M_{z}\right)^{2}+\frac{1}{3} S S_{\mathrm{incoh}}+N N_{\text {incoh }}
\end{aligned}
$$

${ }^{2}$ Ici aussi, pour des raisons de simplicité, nous avons remplacé les carrés des modules par des carrés 


$$
\begin{aligned}
& \sigma^{+-}=\left(M_{x}+\imath M_{y}\right)^{2}+\frac{2}{3} S S_{\text {incoh }} \\
& \sigma^{-+}=\left(M_{x}-\imath M_{y}\right)^{2}+\frac{2}{3} S S_{\text {incoh }}
\end{aligned}
$$

Aussi, pour séparer la partie magnétique de la partie nucléaire de l'intensité diffusée, au lieu de mesurer séparément les intensités 'spin flip' et 'non spin flip', il est en général bien plus précis de choisir une des deux et de la mesurer pour deux directions de la polarisation uniaxiale : la direction verticale (direction $\mathbf{Z}$, voir Figure 4) et la polarisation le long du vecteur de diffusion (direction $\mathbf{X}$ ). La différence entre ces deux mesures donne directement la contribution magnétique cohérente, toutes les contributions incohérentes s'éliminant exactement dans la différence.

$$
\begin{aligned}
& \left(\sigma^{++}\right)_{\mathbf{P} / / \mathbf{Z}}-\left(\sigma^{++}\right)_{\mathbf{P} / / \mathbf{X}=\mathbf{Q}}=\left|M_{Z}\right|^{2} \\
& \left(\sigma^{+-}\right)_{\mathbf{P} / / \mathbf{X}=\mathbf{Q}}-\left(\sigma^{+-}\right)_{\mathbf{P} / / \mathbf{Z}}=\left|M_{Z}\right|^{2}
\end{aligned}
$$

Cette mesure comparative peut se faire aussi bien sur des intensités 'spin flip' que 'non spin flip'. Le résultat est formellement le même. Le choix entre ces deux configurations dépend de la quantité de diffusion incohérente qui existe pour chacune d'entre elles ; les contributions incohérentes s'éliminent dans les différences, mais elles augmentent les incertitudes expérimentales.

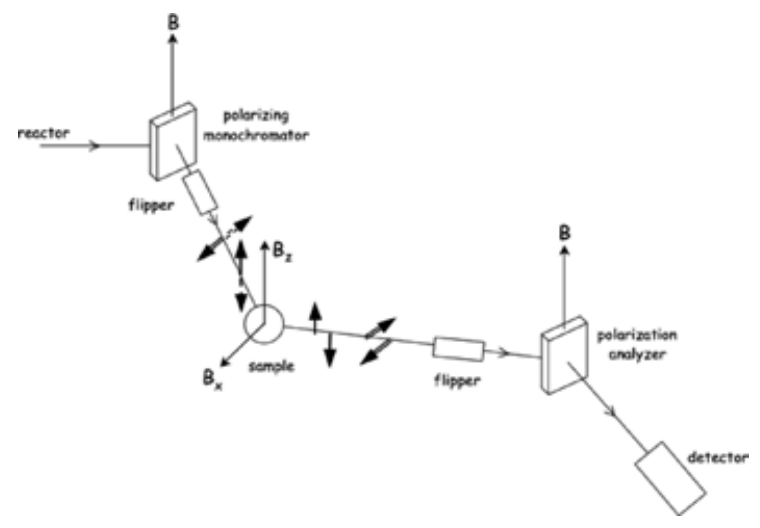

Figure 4. Schéma de principe pour la méthode $X Z$ en analyse de polarisation uniaxiale.

Analyse uniaxiale de la polarisation dans les 3 dimensions [4]

Les formules 4.12 et 4.13 impliquent que l'une des deux mesures soit faite avec la polarisation uniaxiale le long du vecteur de diffusion. Mais il $y$ a des cas où une telle mesure n'est pas possible :

- en diffusion élastique lorsque plusieurs détecteurs sont utilisés en même temps (multicompteur) et collectent des neutrons qui correspondent à des vecteurs de diffusion différents

- en diffusion inélastique quand un détecteur collecte des neutrons qui ont tous le même vecteur d'onde $\mathbf{k}$, mais différents vecteurs de diffusion $\mathbf{Q}$

La méthode $X Y Z$ a été mise au point pour de tels cas [4]. Dans cette méthode on compare, soit dans une configuration 'spin flip' soit dans une configuration 'non spin flip' les intensités mesurées pour 3 directions de la polarisation uniaxiale : une avec la polarisation verticale (direction $\mathbf{Z}$ ) et les 2 autres avec deux directions orthogonales dans le plan horizontal (directions $\mathbf{X}$ et $\mathbf{Y}$ ), comme illustré sur la Figure 5. Ni $X$ ni $Y$ n'est la direction du vecteur de diffusion (direction qui n'est pas forcément unique). 
Cependant, les formules 4.12 et 4.13 peuvent être appliquées (à un coefficient près) en remplaçant les intensités $\mathbf{P} / / \mathbf{Q}$ par une moyenne entre les intensités $\mathbf{P} / / \mathbf{X}$ et $\mathbf{P} / / \mathbf{Y}$.

Pour réaliser de telles mesures la polarisation sur l'échantillon est contrôlée par un système de champs guides : une triple paire de bobines de Helmoltz, une paire selon $\mathbf{X}$, une paire selon $\mathbf{Y}$ et une paire selon $\mathbf{Z}$.

Il est important de noter que cette méthode $X Y Z$, bien qu'elle soit pratiquée dans les 3 dimensions de l'espace, reste néanmoins une méthode d'analyse uniaxiale dans la mesure où l'analyse de la polarisation des neutrons diffusés se fait dans la même direction que la polarisation des neutrons incidents. Un champ magnétique est appliqué sur l'échantillon : la polarisation des neutrons incidents lui est parallèle et pour les neutrons diffusés, seule la composante de la polarisation parallèle à ce champ est analysée.

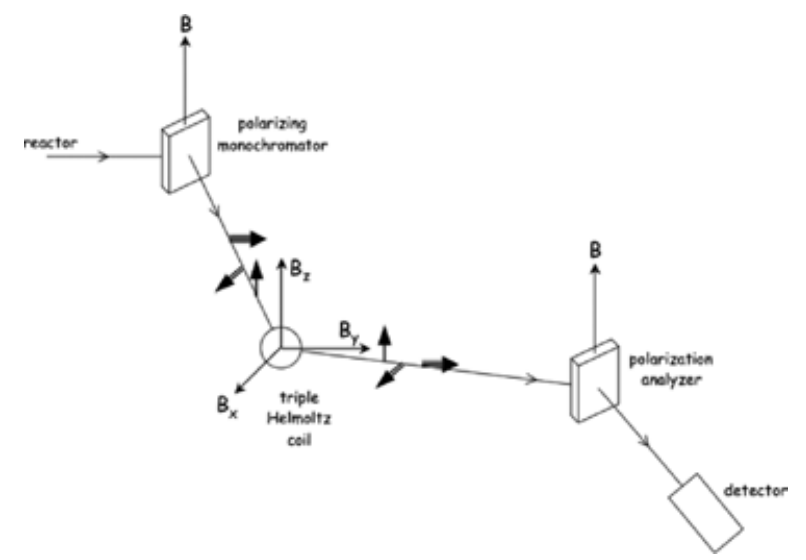

Figure 5. Schéma de principe pour l'analyse uniaxiale de polarisation dans les 3 dimensions.

Analyse sphérique de la polarisation [5]

Dans ce dernier concept, pour chaque direction de la polarisation incidente, la polarisation du faisceau diffusé est analysée dans les 3 directions de l'espace (voir Figure 6). Formellement, pour chaque direction $\mathbf{X}, \mathbf{Y}$ ou $\mathbf{Z}$ de la polarisation incidente, il $y$ a 3 mesures de la polarisation diffusée, soit 9 mesures en tout.

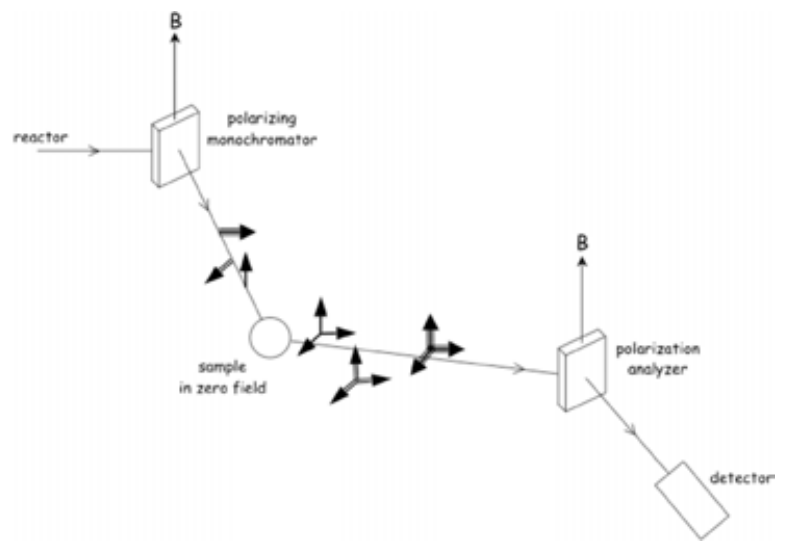

Figure 6. Schéma de principe de l'analyse sphérique de la polarisation [5]. 
La polarisation du faisceau diffusé peut s'écrire comme :

$$
\mathbf{P}^{\prime}=\mathbf{P}_{\mathbf{0}}^{\prime}+\overline{\bar{T}} \mathbf{P}
$$

où le premier terme $\mathbf{P}_{0}^{\prime}$ est une polarisation créée pendant le processus de diffusion et où le deuxième terme $\overline{\bar{T}} \mathbf{P}$ représente une rotation de la polarisation incidente, rotation provoquée par la diffusion. $\overline{\bar{T}}$ est un tenseur $3 \times 3$.

Les 3 composantes du vecteur $\mathbf{P}_{\mathbf{0}}^{\prime}$ peuvent être déterminées directement en mesurant les 3 composantes de la polarisation des neutrons diffusés lorsque le faisceau incident n'est pas polarisé. Les 9 éléments du tenseur $\overline{\bar{T}}$ sont déterminés par les 9 mesures polarimétriques avec des faisceaux incidents polarisés, comme mentionné plus haut.

Dans cette méthode où, pour chaque direction de la polarisation incidente la direction de la polarisation diffusée est analysée dans les 3 directions de l'espace, il est important que la polarisation diffusée ne soit pas perturbée par un champ magnétique qui existerait dans la zone de l'échantillon. C'est pourquoi, dans cette technique, il est important que l'échantillon se trouve dans une zone dépourvue de tout champ magnétique.

\section{Références}

[1] Nathans, R., Shull, C.G., Shirane, G., Andresen, A., J. Phys. Chem. Solids 10 (1959) 138.

[2] Shull, C.G.; Yamada, Y. 1962 J. Phys. Soc. Jap. 17 Suppl. BIII (1962) 1.

[3] Moon, R.M., Riste, T., Koehler, W.C. Phys. Rev. 181 (1969) 920.

[4] Schaerpf, O., Physica B 156-157 (1989) 631, Schaerpf, O., Physica B 182 (1992) 37, Schaerpf, O., Capellmann, H., Phys. Stat. Sol. 135 (1993) 35.

[5] Tasset, F., Physica B 156-157 (1989) 627, Brown, P.J., Forsyth, J.B., Tasset, F., R. Soc. Lond. A442 (1993) 147. 
